By Theorem 2, the solutions of the equation (17) are given by (16). If $x_{i}=\rho_{i}, y_{k}=\sigma_{k}$ is any solution of (13) and we choose $\alpha_{i}=\rho_{i}, \mu_{k}=\sigma_{k}$, $\lambda=f(\rho)$, we have that $s=0$ and the solution becomes $x_{i}=\rho_{i} K^{n-1}$, $y_{k}=\sigma_{k} K^{n+1}$, where $K=A \lambda(A D-B C)$, which is equivalent to the given solution provided $K \neq 0$; that is, provided $x_{i}=\rho_{i}, y_{k}=\sigma_{k}$ is not a solution of (14). It will be noted that if $K \neq 0$, then $t \neq 0$.

Louisiana State University

\title{
A MULTIPLE NULL-CORRESPONDENCE AND A SPACE CREMONA INVOLUTION OF ORDER $2 n-1^{1}$
}

\author{
EDWIN J. PURCELL
}

Part I. A nUll-system $(1, m n, m+n)$ Between the PLanes AND POINTS OF SPACE $(m, n=1,2,3, \cdots)$

1. Introduction. Consider a curve $\delta_{m}$ of order $m$ having $m-1$ points in common with a straight line $d$, and a curve $\delta_{n}{ }^{\prime}$ of order $n$ having $n-1$ points in common with a straight line $d^{\prime},(m, n=1,2,3, \cdots)$. It is assumed for the present that neither $\delta_{m}$ nor $d$ intersects either $\delta_{n}^{\prime}$ or $d^{\prime}$.

In general, through any point $P$ of space there passes one ray $\rho$ which intersects $\delta_{m}$ once and $d$ once, and one ray $\rho^{\prime}$ which intersects $\delta_{n}^{\prime}$ once and $d^{\prime}$ once; $\rho$ and $\rho^{\prime}$ determine a plane $\pi$, the null-plane of $P$. Conversely, a plane $\pi$ determines $m$ rays $\rho_{i}$ and $n$ rays $\rho_{j}^{\prime}$ lying in it which intersect, a ray $\rho$ with a ray $\rho^{\prime}$, in $m n$ points, the null-points of the plane $\pi$.

Any point $\alpha$ in general position determines a ray $\rho$. As $\alpha$ describes a line $l$, the plane $\pi$ of $\rho$ and $l$ contains $n$ rays $\rho^{\prime}$, which intersect $l$ in $n$ points $\beta$; conversely, any point $\beta$ on $l$ determines a ray $\rho^{\prime}$ which determines with $l$ the plane $\pi$, and $\pi$ contains $m$ rays $\rho$ which intersect $l$ in $m$ points $\alpha$-one being the original $\alpha$. Thus an $(m, n)$ correspondence is set up among the points of $l$ with valence zero; there are $m+n$ coincidences and therefore $m+n$ points on any line $l$ whose nullplanes contain $l$.

2. Planes whose null-points behave peculiarly. We can obtain the last result by another method; this will yield additional information about planes whose null-points behave peculiarly.

Let a plane $\pi$ turn about a line $l$ as axis. A ruled surface will be generated by the $m$ rays $\rho_{i}$ lying in $\pi$. This surface is of order $m+1$; $\delta_{m}$ is a onefold curve on the surface and $d$ is an $m$-fold line. Another

${ }^{1}$ Presented to the Society, December 2, 1939. 
ruled surface will be generated in this manner by the rays $\rho_{j}^{\prime}$ lying in $\pi$; its order is $n+1, \delta_{n}^{\prime}$ is a onefold curve and $d^{\prime}$ is an $n$-fold line on this surface. The curve of intersection of these two surfaces is of order $(m+1)(n+1)$ and consists of $l$ and a twisted curve $k_{m n+m+n}$ of order $(m+1)(n+1)-1=m n+m+n$. This $k_{m n+m+n}$ is the locus of the nullpoints of all planes $\pi$ through $l$.

Since a plane $\pi$ meets this in $m n$ points outside $l, k_{m n+m+n}$ must intersect $l$ in $m+n$ points through each of which a ray $\rho$ and a ray $\rho^{\prime}$ pass which are coplanar with $l$. Call such a point on $l, P$. The plane $\rho \rho^{\prime}$ is the null-plane of $P$ and has $m n-1$ null-points outside $l$, and it follows that plane $\rho \rho^{\prime}$ is tangent to $k_{m n+m+n}$ at $P$. The null-planes of the $m+n$ points of intersection of $k_{m n+m+n}$ with $l$ are tangent planes of $k_{m n+m+n}$ at these points.

The line $d$, an $m$-fold line on the first of the two surfaces described above, intersects the second surface in $n+1$ points, which are $m$-fold points on the first surface. The line $d^{\prime}$ intersects the first of the two surfaces in $m+1$ points which are $n$-fold points on the second surface. These points all lie on $k_{m n+m+n}$ and the $m+1$ are $n$-fold points of it and $n+1$ are $m$-fold points of it. $k_{m n+m+n}$ has $m+1 n$-fold points on $d^{\prime}$ and $n+1 m$-fold points on $d$.

$\delta_{m}$ has no actual double points or other multiple points. It is, however, rational and has $(m-1)(m-2) / 2$ apparent double points and its rank is $r=m(m-1)-(m-1)(m-2)=2(m-1)$; that is, the order of its developable surface is $2(m-1)$. Similarly, the order of the developable surface of $\delta_{n}^{\prime}$ is $2(n-1)$. The line $l$ will intersect $2(m-1)$ tangents of $\delta_{m}$ and $2(n-1)$ tangents of $\delta_{n}{ }^{\prime}$. In the plane $\pi$ through $l$ and a tangent line $t$ of the first group, two rays $\rho$ coincide in the line which joins the point of tangency of $t$ with the intersection of $d$ and $\pi$. Of the $m n$ null-points in the plane $\pi, n$ lie on each of the other $m-2$ rays $\rho$, and $2 n$ fall two and two together on the coinciding rays; in these points $k_{m n+m+n}$ is tangent to the plane of $l$ and $t$ and the number of these planes is $2(m+n-2)$.

From the discussion of this section we have the following conclusions:

(1) The planes, $m$ of whose null points coincide with a point of $d$, envelope a surface of class $n+1$; and the planes, $n$ of whose null points coincide with a point of $d$, envelope a surface of class $m+1$.

(2) The planes, $2 n$ of whose null-points coincide two and two on a ray $\rho$, envelope a surface of class $2(m-1)$, $n$ of the remaining null-points lying on each of the other $m-2$ rays $\rho$; the planes, $2 m$ of whose nullpoints coincide two and two on a ray $\rho^{\prime}$, envelope a surface of class 
$2(n-1), m$ of the remaining null-points lying on each of the other $n-2$ rays $\rho^{\prime}$.

Consider a plane $\pi$ through $l$, whose intersection with $d$ is also an intersection with $\delta_{m}$. Call this common point of $d$ and $\delta_{m}, \Delta$. Then the rays $\rho_{i}$ lying in $\pi$ will be the $m-1$ lines joining $\Delta$ to the $m-1$ points of intersection of $\delta_{m}$ and $\pi$, not lying on $d$, and the line $\lambda$ joining $\Delta$ to the intersection of $l$ and the plane of $d$ and the tangent line to $\delta_{m}$ at $\Delta$. This line $\lambda$ will be the limiting position of a ray $\rho$ as a plane revolves about $l$ into the position of $\pi$.

In the osculating planes of $\delta_{m}$ and $\delta_{n}{ }^{\prime}$, three rays coincide. Therefore, in the osculating planes of $\delta_{m}, 3 n$ of the null-points coincide three and three on the triple ray; in the osculating planes of $\delta_{n}^{\prime}, 3 m$ of the nullpoints coincide three and three on the triple ray.

3. Points whose null-planes behave peculiarly. Consider a point $P$ on $d$. The point $P$ determines one $\rho^{\prime}$. Any plane $\pi$ through $\rho^{\prime}$ determines $m$ rays $\rho$ through $P$. Therefore $\pi$ counts $m$ times as null-plane of $P$. Conversely, for every plane through $\rho^{\prime}$ there fall $m$ null-points together at $P$. The surface of class $n+1$ mentioned in $\$ 2$ must have the planes $\pi$ as tangent planes. This surface is a ruled surface consisting of rays $\rho^{\prime}$ which intersect $d$, and conversely. Call this surface $\Sigma$.

The surface formed by rays $\rho^{\prime}$ which intersect a general straight line $l$ is $(\$ 2)$ of order $n+1$, and $d$ intersects this surface in $n+1$ points. Thus there are $n$ rays $\rho^{\prime}$ which intersect $d$ and also an arbitrary line $l$. Therefore the surface $\Sigma$ is of degree $n+1$. The line $d$ is a onefold directrix on $\Sigma_{n+1}$ and $d^{\prime}$ is an $n$-fold directrix; for, the $n$-ic cone of $\delta_{n}^{\prime}$ projected from a point of $d^{\prime}$ will intersect $d$ in $n$ points. The locus of points whose null-planes have $m$ null-points coinciding is $\Sigma_{n+1}$.

Similarly, the ruled surface $\Sigma_{m+1}^{\prime}$ of order $m+1$, consisting of rays $\rho$ that intersect $d^{\prime}$, is the locus of points whose null-planes have $n$ nullpoints coinciding.

Now $\Sigma_{n+1}$ and $\Sigma_{m+1}^{\prime}$ have $m n+1$ generators in common. For the congruence of rays $\rho$ has the characteristic $(1, m)$ and the congruence of rays $\rho^{\prime}$ has the characteristic $(1, n)$ so that, from Halphen's theorem, ${ }^{2}$ there are $1 \cdot 1+m \cdot n=m n+1$ common rays.

Since both rays $\rho$ and $\rho^{\prime}$ through any point on one of these $m n+1$ common rays coincide, any plane through the ray can be taken as null-plane of the point. Every plane of the pencil through any one of the $m n+1$ common rays has $m$ null-points coinciding on $d$ and $n$ nullpoints coinciding on $d^{\prime}$.

\footnotetext{
${ }^{2}$ C. M. Jessop, A Treatise on the Line Complex, 1903, p. 259.
} 
The intersection of $\Sigma_{n+1}$ and $\Sigma_{m+1}^{\prime}$ is of degree $(n+1)(m+1)$. Since $d^{\prime}$ was shown to be an $n$-fold line on $\Sigma_{n+1}$ and is clearly a onefold line on $\Sigma_{m_{+1}^{\prime}}^{\prime}, d^{\prime}$ therefore counts $n$ times in the intersection of these two surfaces. Similarly $d$ counts $m$ times in the intersection. Each of the $m n+1$ common rays of the two congruences counts once in the intersection. The parts just enumerated have total degree $n+m+m n+1$ $=(n+1)(m+1)$. Therefore, the locus of points whose null-planes have $m$ null-points coinciding in one point and $n$ null-points coinciding in another consists of the lines $d$ and $d^{\prime}$ and the $m n+1$ common rays of the two congruences.

Now consider a plane containing $d$; let it intersect $d^{\prime}$ in $D^{\prime}$ and $\delta_{n}^{\prime}$ in $n$ points $N_{i}$. Every point of the $n$ lines $D^{\prime} N_{i}$ is a null-point of this plane-similarly for planes through $d^{\prime}$.

Let point $P$ be on $\delta_{m}$ but not on $d$. One $\rho^{\prime}$ is determined but every line from $P$ to $d$ will be a $\rho$. Therefore, any point of $\delta_{m}$ or $\delta_{n}^{\prime}$ not also a point of $d$ or $d^{\prime}$ has the pencil of planes through the ray of the opposite congruence as null-planes.

\section{Part II. A space CRemona involution of ORDER $2 n-1$ ( $n$ ANY INTEGER)}

4. Definition. Not every skew curve of order $n$ has a secant meeting it in $n-1$ points, and some have only one such secant, but there are also skew curves of order $n$ that have two $(n-1)$-secant lines. In such case they lie on a quadric surface and have a singly infinite system of such secants. The two selected must be two generators of the same regulus.

Consider a fixed twisted curve $\delta_{n}$ of order $n$ having $n-1$ points in common with a fixed line $d$ and $n-1$ points in common with another fixed line $d^{\prime}$. This construction occurs when the two twisted curves $\delta_{n}^{\prime}$ and $\delta_{m}$ in Part I are identical but lines $d$ and $d^{\prime}$ remain skew to each other.

A general point $P$ determines a unique line intersecting $\delta_{n}$ once, at $A$, and $d$ once, at $D$, and a unique line intersecting $\delta_{n}$ once, at $B$, and $d^{\prime}$ once, at $D^{\prime}$. We define $P^{\prime}$, the correspondent of $P$, to be the intersection of lines $A D^{\prime}$ and $B D$. It is an involution.

5. Equations. Let $d$ be $x_{1}=0, x_{2}=0$, and $d^{\prime}$ be $x_{3}=0, x_{4}=0$, and the parametric equations of $\delta_{n}$ be

$$
\begin{array}{ll}
x_{1}=(a s+b t) \prod_{1}^{n-1}\left(t_{i} s-s_{i} t\right), & x_{2}=(c s+d t) \prod_{1}^{n-1}\left(t_{i} s-s_{i} t\right), \\
x_{3}=(e s+f t) \prod_{n}^{2 n-2}\left(t_{i} s-s_{i} t\right), & x_{4}=(g s+h t) \prod_{n}^{2 n-2}\left(t_{i} s-s_{i} t\right),
\end{array}
$$


where $\left(s_{i}, t_{i}\right),(i=1,2, \cdots, n-1)$, are values of the parameter at the $n-1$ points of $\delta_{n}$ on $d$, and for $i=n, n+1, \cdots, 2 n-2$ are values of the parameter at the $n-1$ points of $\delta_{n}$ on $d^{\prime}$. Then the equations of the involution are

$$
\begin{aligned}
& x_{1}^{\prime}=(a d-b c)\left\{(a h-b g) x_{3}-(a f-b e) x_{4}\right\} \prod_{1}^{n-1} \alpha_{i} \prod_{1}^{n-1} \beta_{i}, \\
& x_{2}^{\prime}=(a d-b c)\left\{(c h-d g) x_{3}-(c f-d e) x_{4}\right\} \prod_{1}^{n-1} \alpha_{i} \prod_{1}^{n-1} \beta_{i}, \\
& x_{3}^{\prime}=(f g-e h)\left\{(c f-d e) x_{1}-(a f-b e) x_{2}\right\} \prod_{n}^{2 n-2} \alpha_{i} \prod_{n}^{2 n-2} \beta_{i}, \\
& x_{4}^{\prime}=(f g-e h)\left\{(c h-d g) x_{1}-(a h-b g) x_{2}\right\} \prod_{n}^{2 n-2} \alpha_{i} \prod_{n}^{2 n-2} \beta_{i},
\end{aligned}
$$

where $\alpha_{i} \equiv\left(t_{i} d+s_{i} c\right) x_{1}-\left(t_{i} b+s_{i} a\right) x_{2}$ and $\beta_{i} \equiv\left(t_{i} h+s_{i} g\right) x_{3}-\left(t_{i} b+s_{i} e\right) x_{4}$. It is of order $2 n-1, n$ any integer.

6. The fundamental system. Line $d$ is an $(n-1)$-fold fundamental line of simple contact. The $n-1$ fixed tangent planes through $d$ are $\alpha_{i}=0,(i=1,2, \cdots, n-1)$. The line $d$ is an $F$-line of the first species whose principal surface consists in the $n-1$ planes $\beta_{i}=0$, $(i=1,2, \cdots, n-1)$.

Line $d^{\prime}$ is an $(n-1)$-fold $F$-line of simple contact. The $n-1$ fixed tangent planes through $d^{\prime}$ are $\beta_{i}=0,(i=n, n+1, \cdots, 2 n-2) . d^{\prime}$ is an $F$-line of the first species whose $P$-surface is $\prod_{n}^{2 n-2} \alpha_{i}=0$.

Points $\Delta_{i},(i=1,2, \cdots, n-1)$, intersections of $d$ with $\delta_{n}$ whose parameters on $\delta_{n}$ are $\left(s_{i}, t_{i}\right)$, and points $\Delta_{i}^{\prime},(i=n, n+1, \cdots, 2 n-2)$, intersections of $d^{\prime}$ with $\delta_{n}$, are isolated $n$-fold $F$-points whose $P$-surfaces are, respectively, the above mentioned fixed tangent planes $\alpha_{i}=0,(i=1,2, \cdots, n-1)$, and $\beta_{i}=0,(i=n, n+1, \cdots, 2 n-2)$.

The $(n-1)^{2}$ lines, each joining a $\Delta_{i}$ to a $\Delta_{i}^{\prime}$, are simple $F$-lines without contact. They are $F$-lines of the second species.

The $(n-1)^{2}$ lines of intersection of the fixed tangent planes through $d$ with the fixed tangent planes through $d^{\prime}$ are simple $F$-lines without contact. They are $F$-lines of the second species.

7. Invariant locus. Every point of the curve $\delta_{n}$ is invariant. Every line that intersects $d, d^{\prime}$, and $\delta_{n}$, each once, goes over into itself although it is not pointwise invariant. The locus of these lines is the quadric surface on which $d, d^{\prime}$, and $\delta_{n}$ lie.

8. Intersection of two homaloids. Since they are surfaces of order 
$2 n-1$, two homaloids intersect in a space curve of order $(2 n-1)^{2}$. The fixed part of this curve consists in the lines $d$ and $d^{\prime}$, each counting $n(n-1)$ times, the $(n-1)^{2}$ lines joining the isolated $n$-fold $F$-points of $d$ with those of $d^{\prime}$, each counting once, and the $(n-1)^{2}$ lines of intersection of the fixed tangent planes through $d$ with those through $d^{\prime}$, each counting once. The order of this fixed part is $2 n(n-1)+2(n-1)^{2}$.

The variable part of the curve of intersection is of order $2 n-1$ and corresponds to the line of intersection of the two general planes which go over into the pair of homaloids.

University of ARIzona 